\title{
Electrospun biomaterial scaffolds with varied topographies for neuronal differentiation of human induced pluripotent stem cells
}

\begin{tabular}{|r|l|}
\hline Journal: & Journal of Biomedical Materials Research: Part A \\
\hline Manuscript ID: & Draft \\
\hline Wiley - Manuscript type: & Original Article \\
\hline Complete List of Authors: & $\begin{array}{l}\text { Mohtaram, Nima; University of Victoria, Mechanical Engineering } \\
\text { Ko, Junghyuk; University of Victoria, Mechanical Engineering } \\
\text { King, Craig; University of Victoria, Biomedical Engineering } \\
\text { Sun, Lin; University of Victoria, Medical Sciences } \\
\text { Muller, Nathan; University of Victoria, Mechanical Engineering } \\
\text { Jun, Martin; University of Victoria, Mechanical Engineering Department } \\
\text { Willerth, Stephanie; University of Victoria, Mechanical Engineering }\end{array}$ \\
\hline Keywords: & $\begin{array}{l}\text { human induced pluripotent stem cells, melt electrospinning, solution } \\
\text { electrospinning, scaffold topography, neural tissue engineering }\end{array}$ \\
\hline
\end{tabular}




\title{
Electrospun biomaterial scaffolds with varied topographies for neuronal differentiation of human induced pluripotent stem cells
}

\author{
Nima Khadem Mohtaram ${ }^{1}$, Junghyuk Ko ${ }^{1}$, Craig King ${ }^{2}$, Lin Sun ${ }^{3}$, Nathan Muller ${ }^{1}$, Martin \\ Byung-Guk Jun ${ }^{1}$ and Stephanie M. Willerth ${ }^{1,3,4, *}$ \\ ${ }^{1}$ Department of Mechanical Engineering, University of Victoria. PO Box 1700, STN CSC, \\ Victoria, BC V8W 2Y2, Canada. \\ ${ }^{2}$ Department of Biomedical Engineering, University of Victoria. PO Box 1700, STN CSC, \\ Victoria, BC V8W 2Y2, Canada \\ ${ }^{3}$ Division of Medical Sciences, University of Victoria. PO Box 1700, STN CSC, Victoria, BC \\ V8W 2Y2, Canada \\ ${ }^{4}$ International Collaboration on Repair Discoveries (ICORD), Vancouver, BC V5Z 1M9, Canada \\ * Author to whom all correspondence should be addressed. \\ *Contact Information: \\ Dr. Stephanie Willerth \\ Department of Mechanical Engineering and Division of Medical Sciences \\ University of Victoria \\ PO Box 1700 STN CSC \\ Victoria, BC V8W 2Y2, Canada \\ Email: willerth@uvic.ca. \\ Phone: +1 (250) 7217303 \\ Fax: +1 (250) 7216051
}




\begin{abstract}
In this study, we investigated the effect of micro and nanoscale scaffold topography on promoting neuronal differentiation of human induced pluripotent stem cells (iPSCs) and directing the resulting neuronal outgrowth in an organized manner. We used melt electrospinning to fabricate poly ( $\varepsilon$-caprolactone) (PCL) scaffolds with loop mesh and biaxial aligned microscale topographies. Biaxial aligned microscale scaffolds were further functionalized with retinoic acid releasing PCL nanofibers using solution electrospinning. These scaffolds were then seeded with neural progenitors derived from human iPSCs. We found that smaller diameter loop mesh scaffolds $(43.7 \pm 3.9 \mu \mathrm{m})$ induced higher expression of the neural markers Nestin and Pax6 compared to thicker diameter loop mesh scaffolds $(85 \pm 4 \mu \mathrm{m})$. The loop mesh and biaxial aligned scaffolds guided the neurite outgrowth of human iPSCs along the topographical features with the maximum neurite length of these cells being longer on the biaxial aligned scaffolds. Finally, our novel bimodal scaffolds also supported the neuronal differentiation of human iPSCs as they presented both physical and chemical cues to these cells, encouraging their differentiation. These results give insight into how physical and chemical cues can be used to engineer neural tissue.
\end{abstract}

Keywords: human induced pluripotent stem cells, melt electrospinning, solution electrospinning, scaffold topography, neural tissue engineering 


\section{INTRODUCTION}

Human pluripotent stem cells (PSCs) can become any cell type found in the body, making them a powerful tool for engineering tissues. Additionally, PSCs, including embryonic stem cells (ESCs) and induced pluripotent stem cells (iPSCs), continuously self-renew, enabling the generation of large quantities of cells for transplantation $[1,2]$. Human iPSCs are generated from somatic cells, such as fibroblasts, which are reprogrammed by introducing transcription factors that cause the cells to function like ESCs. One advantage of using human iPSCs instead of human ESCs is that such cell lines could be generated from patients, reducing the probability of immune rejection. The use of iPSCs also avoids the ethical issues associated with using embryos to derive human ESC lines [3,4].

Many studies have differentiated human iPSCs into neural phenotypes for a variety of applications [5-12]. The Li group showed that transplanted neural crest cells derived from human iPSCs promoted accelerated regeneration of the sciatic nerve in a rat injury model [13]. They also observed no tumor formation for a 1 year after transplantation of these cells. Neurospheres derived from human iPSCs have been transplanted into the spinal cord injury of mice models where they differentiated into three major neural lineages, neurons, astrocytes, and oligodendrocytes and promoted a significant increase in functional recovery compared to control animals [8]. Another study differentiated human iPSCs into neural crest cells in vitro and then transplanted these cells into a fetal lamb model of spinal cord injury [14]. These cells survived and differentiated into neurons after transplantation with no tumor formation observed. These studies demonstrate the potential of using human iPSC-derived neural cells for treating different types of injuries to the nervous system. 
One of the major challenges when using human iPSCs for tissue engineering applications is how to control the differentiation process to produce three dimensional structures similar to those found in healthy tissue. Stem cell behavior can be significantly modulated by mechanical and topographical physical cues [15-18]. This area remains relatively understudied compared to the wide body of literature describing the use of chemical cues for promoting stem cell culture and differentiation. For example, the differentiation rate of human ESCs seeded on salt leaching fabricated porous scaffolds increased with scaffold stiffness [19]. The biophysical microenvironment also influences the rate of reprogramming of somatic cells into iPSCs [18]. Scaffold topography can also control the differentiation of PSCs into specific phenotypes [16, 20, 21]. For instance, the topography of nano/microstructured scaffolds influences the differentiation of human iPSCs into neuronal lineages [16]. Using silicon grating structures with different widths, they found that nanoscale structures induced an up regulation in the expression of neuronal markers in human iPSCs compared to microscale structures, indicating the influence of nano topography on the induction of neuronal lineage.

Electrospun fibers have been evaluated for different neural tissue engineering applications as they can present both chemical and physical cues [22-28]. Xie et al. showed aligned nanofibers reduce the fraction of cells that differentiated into astrocytes, which are undesirable when treating spinal cord injury [20]. The same study reported that mouse ESC-derived neurons seeded on the aligned fibers showed longer neurite outgrowth compared to the same cells seeded on the randomly-oriented nanofibers. Other researchers have shown that seeding human ESCderived neural cells onto aligned nanofibers enhanced neuronal differentiation compared to randomly orientated nanofibers [26]. Additionally, small molecules and growth factors can be encapsulated inside of such nanofiber scaffolds [24, 27-29]. For instance, Madduri et al. used 
electrospun nanofibers to encapsulate glial cell line-derived neurotrophic factor (GDNF) and nerve growth factor (NGF) with varied topographies to develop multifunctional scaffolds for peripheral nerve regeneration [24]. In another study, retinoic acid (RA) releasing nanofibers enhanced the differentiation of mesenchymal stem cells into neural lineages [27]. In our previous study, controlled release of RA from PCL nanofibers was provided over one month and such scaffolds were able to promote the neuronal differentiation of mouse iPSCs [28]. Overall, studies on electrospun fibers continue to play a key role in the neural tissue engineering and controlled release of neural drugs for the development of on-going strategies for clinical applications.

These aforementioned studies all used solution electrospinning to fabricate such scaffolds. However, melt electrospinning provides a better way to control the topography of fibrous scaffolds [6, 30-33]. The process of melt electrospinning involves melting a polymer to generate flow for producing fibers with a high degree of reproducibility compared to the more commonly used solution electrospinning. Melt electrospinning does not require a solvent whereas most solvents used in solution electrospinning are cytotoxic, providing an additional key advantage. This technique serves as a powerful tool for fabricating cell invasive scaffolds with varied architecture for tissue engineering applications [33, 34]. The successful attachment and cell viability of fibroblast cells seeded on PCL melt electrospun scaffolds has been reported [34]. Also, our group recently reported that melt electrospun PCL scaffolds could support the neuronal differentiation of murine ESCs [33]. The topography of scaffolds can be custom tailored by the tuning of parameters such as flow rate, applied voltage, nozzle size and spinning temperature $[29,30,33,35]$.

In order to successfully translate PSC-based engineered tissues for clinical applications, the culture and differentiation methods should be reproducible and avoid the use of animal products 
as they can induce immune responses post transplantation [36-38]. For this study, we cultured our human iPSCs in completely defined conditions to maintain their levels of pluripotency [36, 38]. These undifferentiated iPSCs were seeded into microwells in the presence of chemically defined neural induction media to form neural aggregates with uniform diameters, increasing the reproducibility of this process compared to tradition methods for forming embryoid bodies [37]. Thus, both our iPSC-derived neural progenitors and our fibrous scaffolds would be suitable for further translation in terms of pre-clinical and clinical studies.

In this work, we investigated how the topography of PCL melt electrospun scaffolds, including loop mesh and biaxial aligned topographies, influenced the differentiation of these human iPSC-derived neural aggregates into neurons and the resulting neurite outgrowth. Due to its low melting point $\left(\sim 60^{\circ} \mathrm{C}\right)$, PCL can be easily electrospun into varied topographies $[32,33]$. It is also a biocompatible polymer that can serve as a scaffold for stem cell culture [20, 28, 33]. We have chosen to focus on generating neurons due to their therapeutic potential for the treatment of spinal cord injuries [39]. We studied the effects of fiber diameter on neuronal differentiation of human iPSCs using two different fiber diameters in the loop mesh scaffolds. Additionally, we also successfully engineered scaffolds consisting of biaxial aligned microfibers, for further investigating the effect of physical cues on the neuronal differentiation of human iPSCs. These scaffolds were also functionalized with RA-releasing nanofibers as well to determine if the nanofibers also had an influence on the resulting stem cell behavior. This combination of encapsulated nanofibers with biaxial aligned PCL scaffolds also supported the differentiation of human iPSCs. Our combination of human iPSC-derived neural progenitors and these novel tissue-engineered scaffolds could serve as a new strategy for neural tissue engineering applications. 


\section{MATERIALS AND METHODS}

\section{Melt and solution electrospinning setup}

Poly ( $\varepsilon$-caprolactone) (PCL, average $\left.\mathrm{M}_{\mathrm{n}} \sim 45,000\right)$ and retinoic acid (RA) (all-trans, $\geq 98 \%$ HPLC, powder) were acquired from Sigma-Aldrich Corporation (St. Louis, MO, USA). Dichloromethane (DCM) (reagent/ACS grade) and methanol were purchased from VWR International (Edmonton, AB, Canada). The melt and solution electrospinning setups used for the fabrication of microfibers and encapsulated nanofibers were previously reported [28, 33]. Scaffolds with loop mesh morphology were fabricated by melt electrospinning using $200 \mu \mathrm{m}$ and $500 \mu \mathrm{m}$ nozzle sizes and hencefore shall be referred to as loop mesh 200 and loop mesh 500 respectively. We had showed previously that fiber diameter increased with the nozzle diameter, enabling fabrication of scaffolds with different fiber diameters [33]. Biaxial aligned scaffolds were fabricated using a $200 \mu \mathrm{m}$ nozzle for fiber extrusion. Bimodal scaffolds were fabricated by using solution electrospinning to overlay nanofibers containing RA over the biaxial aligned microfibers. The details of electrospinning parameters for each topography are given in Table 1. The composition of our $0.2 \%(w / v)$ PCL-RA solution was previously reported [28].

\section{Micro and nanostructure characterization}

Characterization of topography was performed using a cold emission Hitachi S-4800 FE scanning electron microscopy (SEM) machine to image all scaffolds at low and high magnification. The details of SEM protocol were reported [28, 33]. For each scaffold, three distinct images were captured at 30x magnification with 50 fibers measured to determine the average fiber diameter of each scaffold. For nanofiber characterization, images were captured at 10,000x magnification. 


\section{Human iPSCs culture and formation of neural aggregates}

All reagents were purchased from STEMCELL Technologies ${ }^{\mathrm{TM}}$ unless otherwise specified. Human iPSCs (the 1-MCB-01 line) generated from human foreskin cells were received from the WiCell Research Institute [3]. Human iPSCs were cultured at $37^{\circ} \mathrm{C}$ and $5 \% \mathrm{CO}_{2}$ on Vitronectin XFTM coated surfaces [36]. To maintain pluripotency, cells were cultured in the presence of TeSR $^{\text {TM}}-E 8^{\text {TM }}$ media in 6 well plates [38]. Undifferentiated human iPSCs were dissociated into a single cell solution using Gentle Cell Dissociation Reagent (STEMCELL Technologies ${ }^{\mathrm{TM}}$ ), which was then distributed into a single well of an Aggrewell ${ }^{\mathrm{TM}} 800$ plate in the presence of 2 mL of STEMdiff ${ }^{\text {TM }}$ Neural Induction Medium (NIM, STEMCELL Technologies) (STEMCELL Technologies $^{\mathrm{TM}}$ ) [37]. These plates enable formation of consistent, neural aggregates containing 4000-5000 cells. $1.5 \mathrm{~mL}$ of media was replaced with fresh NIM daily.

\section{Neural progenitor cell seeding onto scaffolds}

After 5 days, the aggregates containing neural progenitor cells were seeded onto the scaffolds. Approximately 4 neural aggregates were seeded into each well of the 6-well plates containing loop mesh or biaxial aligned or bimodal scaffolds. $1 \mathrm{~mL}$ of NIM was added to each well and the cultures and scaffolds were incubated at $5 \% \mathrm{CO}_{2}$ and $37^{\circ} \mathrm{C}$ for 12 days.

\section{Cell viability and immunohistochemistry analysis}

The viability of human neural aggregates seeded on the PCL scaffolds was analyzed qualitatively after 12 days by using a LIVE/DEAD® Viability/Cytotoxicity Kit (Invitrogen). The details of our protocol have been previously published [20, 33, 40]. Briefly, 12-day-old neural aggregates grown on the scaffolds were treated with calcein AM, which is enzymatically converted to green fluorescing calcein by the naturally present intracellular esterase activity in live cells. They were also treated with a stain for cytotoxicy, ethidium homodimer-1, which 
fluoresces red upon binding to nucleic acids accessed through the ruptured cell membranes of dead cells. Media was first removed and then each well was gently washed twice with Dulbecco's phosphate buffered saline (D-PBS) (Invitrogen). Diluted stain solution was then added to each well of a 6-well-plate and the cells were incubated at room temperature. After 45 minutes, we then imaged each well by using IncuCyte ${ }^{\circledR}$ ZOOM Essen BioScience ${ }^{\circledR}$ fluorescent microscope and LEICA 3000B inverted microscope containing an X-cite series 120Q fluorescent light source (Lumen Dynamics) coupled with a Retiga 2000R fast-cooled mono 12-bit camera (Q-imaging). Quantitatively, an IncuteCyte ${ }^{\circledR}$ ZOOM Essen BioScience ${ }^{\circledR}$ fluorescent microscope was used to measure the green fluorescent intensity in each image, corresponding to the percentage of viable cells present. Three distinct cell/scaffold images were selected per scaffold for analysis using the IncuteCyte ${ }^{\circledR}$ ZOOM Fluorescent Processing Software.

We qualitatively assessed the differentiation of human iPSCs using immunocytochemistry to detect the neuron-specific protein $\beta$-III-tubulin as previously reported [20, 33, 40]. Briefly, differentiated cells were fixed with a $10 \%$ formalin solution (Sigma-Aldrich, St. Louis, MO, USA) for 1 hour at room temperature and then permeabilized with $0.1 \%$ Triton-X solution (Sigma-Aldrich, St. Louis, MO, USA). Wells then were blocked with 5\% normal goat serum (NGS, Millipore) at $2-8{ }^{\circ} \mathrm{C}$ for 2 hours. The primary antibody for $\beta$-III-tubulin (Millipore, 1:500 dilution) was added to each well. Three washes with PBS were performed and the Alexafluor488-conjugated secondary antibody was added and incubated for 4 hours. After an additional set of washes, images were captured at $515 \mathrm{~nm}$ for green fluorescence.

\section{Quantitative analysis of neurite extension and cell-body cluster area}

An IncuCyte ${ }$ ZOOM Essen BioScience ${ }^{\circledR}$ fluorescent microscope was used to analyze fluorescent images of Tuj-1 stained neural progenitors seeded on all sets of scaffolds at $20 \mathrm{X}$ 
magnification. Three EBs per scaffold were selected per group and 14 stacks of images were collected for analysis using the IncuCyte ${ }^{\circledR}$ ZOOM NeuroTrack Software ${ }^{\mathrm{TM}}$. Maximum neurite length and cell body cluster area of neural progenitors were calculated for each neural progenitor per scaffold. The maximum neurite length is defined by the length of the longest neurite that extended from the neural aggregate. The cell-body cluster area is the size of the neural aggregates and their extended neurites after 12 days of culture.

\section{Real time quantitative polymerase chain reaction (qPCR) analysis}

For each set of scaffolds, total RNA was extracted from cultures using an RNeasy kit (Qiagen), and cDNA was synthesized from $1 \mu \mathrm{g}$ of total RNA using a High-Capacity cDNA Reverse Transcription Kit (Life Technologies). Quantitative real-time polymerase chain reaction (qPCR) was then performed with $50 \mathrm{ng}$ of the reversely transcribed cDNA through the comparative $\mathrm{Ct}$ method and by using fast mode in the StepOnePlus ${ }^{\mathrm{TM}}$ Real-Time PCR System (Life Technologies), employing TaqMan® Fast Advanced Master mix and TaqMan® Gene Expression Assays (Life Technologies). Real time qPCR analysis was done to study the expression of Oct4 pluripotency marker, Lin28 (expressed by undifferentiated human embryonic stem cells), Nestin (expressed by neural stem/progenitor cells), and Pax6 (a neuroectoderm marker for human pluripotent stem cells) markers. For the relative quantification of the target gene expression, cycle threshold $\left(\mathrm{C}_{\mathrm{t}}\right)$ values of the target genes were normalized against that of the endogenous housekeeping gene, 18S rRNA. $\Delta \Delta \mathrm{C}_{\mathrm{t}}\left(=\Delta \mathrm{C}_{\mathrm{t}}\right.$ sample (differentiated cells) $-\Delta \mathrm{C}_{\mathrm{t}}$ reference (undifferentiated human iPSCs)) values were plotted as relative levels of gene expression and the data is reported as mean \pm standard error of the mean. 


\section{Statistical analysis}

Data are presented as mean values \pm standard deviation of the mean except where previously stated. Statistical analysis was performed using STATISTICA 9 by applying a standard $t$-test to compare data between groups. Significance was considered at the $\mathrm{p}<0.05$ level.

\section{RESULTS}

\section{Topographical characterization of scaffolds}

We fabricated loop mesh 200, loop mesh 500, biaxial aligned scaffolds and bimodal scaffolds using melt and solution electrospinning. As mentioned in the methods, the 200 and 500 refer to the diameter of the nozzles in $\mu \mathrm{m}$ used to fabricate each set of scaffolds. The loop mesh 200 scaffolds had an average fiber diameter of $43.7 \pm 3.9 \mu \mathrm{m}$. Loop mesh 500 scaffolds had an average fiber diameter of $85 \pm 4 \mu \mathrm{m}(n=50)$. Low and high magnification SEM images of both sets of loop mesh scaffolds are shown in Figures 1A, 1B, 1C and 1D. Loop mesh scaffolds have a controllable porosity and fiber diameter, and were previously shown to be capable of supporting mouse ESC differentiation into neurons [33]. Figures $1 \mathrm{E}$ and $1 \mathrm{~F}$ show the topography of the biaxial aligned electrospun PCL microfibers fabricated by using $200 \mu \mathrm{m}$ nozzle tip diameter. Highly aligned and stretched microfibers can be fabricated if the speed is set to be faster than the depositing viscous jet. The biaxial aligned scaffold consisted of 20 layers of evenly spaced and fully aligned microfibers. For biaxial aligned scaffolds, the average fiber diameter was measured as $42.3 \pm 2.78 \mu \mathrm{m}(n=50)$. From these measurements, the average separation distance, the distance between two individual fibers, for each scaffold was calculated (Table 2). Fabrication of biaxial aligned scaffolds with different diameters was not possible as the microfibers did not attach to each other when the fiber diameter increased. Figure $1 \mathrm{G}$ shows 
the topography of our novel bimodal scaffolds. Our previous study showed that successful encapsulation of RA inside PCL nanofibers led to month long controlled release of RA [28]. Since biaxial aligned PCL microfibers are porous in nature, RA-encapsulated PCL nanofibers were spun on such structures to increase the efficiency of cell adhesion by increasing the surface area for migrating cells, allowing them to adhere to the surface of fibers in such scaffolds. Figure $1 \mathrm{H}$ shows the topography of well-aligned RA-encapsulated PCL nanofibers that have been stretched and parallelized by being spun between biaxial aligned PCL microfibers. The average fiber diameter for RA encapsulated PCL nanofibers was measured as $344.9 \pm 33.6 \mathrm{~nm}(n=100)$.

\section{The effect of loop mesh topography on the behavior of human iPSC-derived neural progenitors}

Neural aggregates containing human iPSC-derived neural progenitor cells were cultured on both sets of loop mesh scaffolds for 12 days. Figure $2 \mathrm{~A}$ and $2 \mathrm{~B}$ show the bright field and live/dead images of seeded cells onto loop mesh 200 scaffolds. The neural progenitors were able to adhere to these scaffolds and migrate along the fibers. After 12 days, these neural progenitors displayed high levels of viability when seeded upon loop mesh scaffolds (Figure 2B). A subset of the seeded cells stained positive for the neuronal marker Tuj1, indicated successful differentiation into neurons (Figure 2D). After 12 days, the cells had started to migrate outward from the spherical neural aggregate along the looped fibers, with distance of migration dependent on the scaffold morphology. Bright field, live/dead, and immunocytochemistry images of cells seeded on loop mesh 500 can be seen in Figures 2E, 2F, 2G, and 2H, respectively. Compared to the loop mesh 200 scaffolds, the cells were not elongated, possibly due to the thick fibers acting as an obstacle to migration. The cells are located in the gap between of fibers (Figure $2 \mathrm{G}$ ) and tend not to differentiate or migrate out in any particular fiber direction. In fact, the human iPSC- 
derived neural aggregates did not respond strongly to loop mesh 500 scaffolds. In contrast, human iPSC-derived neural progenitor seeded on loop mesh 200 scaffolds were oriented and elongated along fibers (Figure 2D). These results suggest that the loop mesh 200 scaffolds served as a better substrate for human iPSC-derived neural progenitors than the loop mesh 500 scaffolds.

\section{Biaxial aligned and bimodal scaffolds}

Both biaxial aligned and bimodal scaffolds were able to support the culture and differentiation of human iPSC-derived neurons (Figure 3 and 4). The live/dead images demonstrate that biaxial aligned scaffold topographies are viable substrates for human iPSCs because the majority of the seeded cells fluoresced green (Figure 3B). Figure 3D shows that scaffold topography serves to direct neurite extension as seen by the large number of cells staining positive for the neuronal marker Tuj1 that have migrated along the microscale structures. The neurons extend along the fibers of biaxial aligned scaffolds and there appears to be regions of dense cell growth, which shows the areas that have the most extracellular support. As can be seen (Figure 3D), neural progenitors of human iPSCs were elongated along the axis of microstructured PCL biaxial aligned scaffolds. This cell behaviour was totally dependent on topography as the neurite outgrowth correlated with fiber direction. Figures 4A and 4B show that the cells had properly attached to the bimodal scaffolds. The interactions between cells into bimodal scaffolds can be seen in Figure 4C and 4D. Figure 4C and 4D show the neuronal differentiation of single neural progenitors seeded on our scaffolds. Figure 4E and 4F show the interactions of two human iPSC-derived neural progenitors. For these scaffolds, the neurites extend in all directions, possibly due to the presence of the nanofibers. 


\section{Quantitative analysis of cell viability, neurite outgrowth and differentiation}

Figure 5A shows the intensity of green fluorescence (representing \% of live cells in each respective neural aggregate) for all cultures. Loop mesh 200 and 500 scaffolds displayed the intensity of green fluorescence to be $81 \pm 8 \%$ and $70 \pm 1 \%$ respectively. After 12 days of seeding, the intensity of green fluorescence was $87.2 \pm 6 \%$ for the neural progenitors seeded onto biaxial aligned scaffolds. The intensity of green fluorescence was $95.1 \pm 2 \%$ for the neural progenitors seeded onto bimodal scaffolds. The neural progenitors seeded onto bimodal scaffolds displayed the highest levels of viability when compared to all types of scaffolds fabricated. Cell body cluster area and maximum neurite field were calculated for each neural progenitor seeded on loop mesh 200, loop mesh 500, biaxial aligned and bimodal scaffolds (Figure 5B and 5C). As it can be seen from Figure 5B, the neural progenitors cultured on loop mesh 200 scaffolds exhibited a higher cluster area compared to those seeded on loop mesh 500 scaffolds, but no significant difference was observed in terms of maximum neurite extension (Figure 5C). The cell body cluster area of neural progenitors cultured on biaxial aligned samples was larger than that of neural progenitors seeded on bimodal scaffolds. The maximum neurite length for neural progenitors seeded on biaxial aligned scaffolds was also longer $(\sim 280 \%)$ compared to the cells seeded on the other scaffold types, showing that the biaxial aligned scaffolds enhanced the neurite outgrowth of neural progenitors compared to other scaffolds. Significantly, such scaffolds also showed the maximum cell body cluster area and the maximum neurite length compared to other type scaffolds. Overall, these results suggest that biaxial aligned scaffolds would serve as the best substrate for promoting spreading and guidance of differentiated cells.

Quantitative polymerase chain reaction was used to examine the gene expression levels of the following markers: Oct4 (transcription factor associated with the self-renewal of pluripotent 
stem cells), Lin28 (microRNA involved in self-renewal of pluripotent stem cells), Nestin (cytoskeletal protein expressed by neural stem/progenitor cell), and Pax6 (protein that regulates the development of neuroectoderm marker). The mRNA expression of Oct4 (2.6-7.2 fold change) and Lin28 (2.5-5.9 fold change) decreased, and the expression of Pax6 (12.0-16.2 fold change) and Nestin (2.0-3.0 fold change) increased in the differentiated cells (12 days) compared to undifferentiated cells. The decreased level of the pluripotency markers is expected as the cells differentiate into mature phenotypes. The expression of Oct4 for cells seeded on biaxial aligned scaffolds was decreased compared to the cells cultured on other scaffolds. There was also a significant difference between loop mesh 200 and other scaffolds for the expression of Oct4. Nestin is expressed much earlier than Pax6, increases in Pax6 expression suggest the cells are further along in the differentiation process. The levels of nestin expressed were similar between biaxial aligned and bimodal scaffolds. However, Pax6 expression was increased in the cells seeded on the biaxial aligned scaffolds, indicating these scaffolds further enhanced differentiation. The cells cultured on loop mesh 200 scaffolds showed $11.2 \%$ more Pax6 marker expression compared to loop mesh 500 scaffolds, indicating that fiber diameter influenced the neural differentiation of the cells. These results suggest that the orientation of microfibers and their average diameter influenced the differentiation of human iPSCs through mechanical cues.

\section{DISCUSSION}

The major advantage of melt electrospun scaffolds for tissue engineering applications is the level of control and reproducibility that can be achieved in terms of fiber diameter and topography. Melt electrospinning can be used as a powerful fabrication technique to construct various structures while controlling their topography with an excellent degree of repeatability[28, 30, 31, 33-35]. We have previously used melt electrospinning to fabricate loop mesh scaffolds 
with varied topographical and mechanical properties [41]. Such loop mesh scaffolds have been shown to be an excellent substrate to support the attachment and neuronal differentiation of neural progenitors derived from murine ESCs. Based on these promising results, we investigated the effect of such topographical properties on the neuronal differentiation of human iPSCs. We seeded human iPSC-derived neural aggregates onto loop mesh scaffolds with different fiber diameter, where they could survive, migrate, and differentiate into neurons. The cells cultured on loop mesh 200 scaffolds were able to migrate along the fiber. Since the loop mesh 200 scaffolds served as a better substrate for human iPSC culture, we used the $200 \mu \mathrm{m}$ nozzle size and increased the speed of the melt deposition from 200 to $1700 \mathrm{~mm} / \mathrm{s}$, to fix the fiber diameter $(\sim 45$ $\mu \mathrm{m})$ and to control the alignment of scaffolds while converting the topography from loop mesh to highly aligned biaxial scaffolds. In addition to the loop mesh scaffolds, we also successfully designed and tested the biaxial aligned scaffold topography in order to report its promising ability to provide physical cues for the differentiation of pluripotent stem cells.

Although using melt electrospinning for drug delivery applications has been reported [42], it is not commonly used to produce controlled release of drugs from fibrous scaffolds unlike solution electrospinning. Therefore, many groups are still using solution electrospinning to encapsulate bioactive agents into nanofibers for neural tissue engineering applications [27-29]. Here, we combined the advantages of both techniques, by using the control over topographical features offered by melt electrospun microfibers and the encapsulation of drugs and controlled release offered by solution electrospun nanofibers.

PSCs have previously been shown to respond to various physical cues [16, 20, 26, 28]. In this work, we studied how human iPSCs seeded onto electrospun scaffolds would respond to changes in micro and nanotoporaphy features. Here, on loop mesh 200 scaffolds $(43.7 \pm 3.9 \mu \mathrm{m})$, human 
iPSCs were well elongated and migrated outward from the neural aggregates along the fibers direction (Figure 2D) while cells have shown to be located between the gap of fibers when seeded onto loop mesh 500 scaffolds $(85 \pm 4 \mu \mathrm{m})$ without any migration, indicating how decreasing fiber diameter could enhance the neuronal differentiation of such cells. Similar to our results, Pan et al have shown that the morphology of human iPSCs had been strongly structure dependent, and they have qualitatively and quantitatively shown that the cells seeded on the smallest topography (on a $350 \mathrm{~nm}$ substrate) had the highest neuronal differentiation [16]. In addition to fiber diameter, our novel biaxial aligned scaffolds also demonstrated that the orientation of fibers could strongly control the direction or neurites outgrowth from human iPSCs. When the neural progenitor cells derived from human iPSCs were seeded on the biaxial aligned scaffolds, the cells drastically aligned and elongated along the direction of the microstructure. In terms of nanoscale topography, aligned electrospun nanofiber scaffolds have previously shown to guide the neurite outgrowth from mouse ESCs and iPSCs when seeded on such scaffolds [20, 28]. Mahairaki et al. have showed that the human ESC-derived neural precursors cultured on aligned micro- and nanofibers had shown a higher rate of neuronal differentiation than other random micro and nanofibers [26]. We have previously shown that interaction with the physical cues influenced cell behaviour as aligned topographies directed the outgrowth of neurons from mouse iPSCs [28]. To gain further insight in how these microscales cues are influencing differentiation, flow cytometry should be also used to quantify the presence of all the differentiated neural phenotypes on our current scaffolds.

Neural progenitor cells derived from human iPSCs cultured on biaxial aligned microfiber scaffolds demonstrated the longest degree of neurite outgrowth $(\sim 573 \mu \mathrm{m})$ compared to those seeded onto other scaffolds. Outgrowth of human iPSCs-derived neurons has been demonstrated 
to be strongly directed by physical cues including the orientation of fibers along with the size of the fibers. Although, our present study showed that the biaxial aligned microfiber scaffolds could enhance the differentiation of cells into neurons, the maximum neurite outgrowth $(\sim 573 \mu \mathrm{m})$ is still smaller than previous findings for the maximum neurite length $(\sim 1600 \mu \mathrm{m})$ which were reported for mouse ESCs seeded onto aligned nanofibers [20]. This could be due to the fact that mouse cultures were incubated for longer time ( 8 days for embryoid body formation followed by 14 days of culture) compared to our human cultures ( 5 days for aggregate formation followed by 12 days of culture). On the other hand, studies on nanofiber scaffolds fabricated using solution electrospinning have been limited to only randomly-oriented and aligned topographies with very low degree of repeatability and controllability, making it hard to produce other topographies such as biaxial aligned scaffolds, loop mesh and other possible designs. However, decreasing the fiber diameter to nanoscale might be possible by tuning the melt electrospinning parameters, allowing fabrication of biaxial aligned nanofiber scaffolds to further promote the maximum neurite length of seeded neural progenitor cells derived from human iPSCs.

We reported that microscale topography could significantly promote the expression of neural marker Pax6 in our human iPSC-derived neural progenitors. These results indicate that the melt electrospun topography induced the expression of neural markers in these cells. Human iPSCderived neural progenitors showed the highest expression of Pax6. However, the cells cultured on the bimodal scaffolds had higher rates of Pax6 expression compared to all loop mesh scaffolds. Our bimodal scaffolds were fabricated in order to determine if PCL nanofibers encapsulating RA would assist the iPSCs in further expression of neural marker Pax6. Compared to biaxial scaffolds without any RA nanofibers, the bimodal scaffolds showed that the iPSCs expressed less Pax6 and therefore bimodal scaffolds show no advantage over only biaxial 
aligned scaffolds. However, our bimodal scaffolds could support the interactions of two human iPSC-derived neural progenitors and such scaffolds demonstrated the highest percentage of viable cells $(\sim 95 \%)$ compared to the other scaffolds which could be due to the presence of bioactive nanofibers filling the gap between blank PCL microfibers.

We have shown that human iPSCs can survive and differentiate into neurons when seeded onto fibrous melt electrospun and bimodal PCL scaffolds. We investigated the physical cues of electrospun microfiber scaffolds on the neuronal differentiation of human iPSCs. All loop mesh, biaxial aligned and bimodal scaffolds supported neuronal differentiation as the seeded cells expressed the neuron specific protein $\beta$-III-tubulin. Particularly, neurite outgrowth was directed by the scaffold topography of our biaxial aligned scaffolds. Our scaffolds and cell culture methods are completely chemically defined, making our strategy a promising approach for clinical neural tissue engineering applications. In this study, we focused mainly on generating neurons as they promote regeneration. In the future, we will conduct longer studies and collect quantitative data could yield a greater understanding of the mechanisms behind how human iPSCs differentiate into neurons. Additionally, we will aim to encapsulate larger molecules such as growth factors, like GDNF, inside bimodal scaffolds to further enhance neuronal differentiation of human iPSCs. Overall, this study demonstrated how topography can be used to differentiate human iPSC-derived neural progenitors into neurons while being able to direct neurite outgrowth as well. 


\section{ACKNOWLEGMENTS}

The authors would like to acknowledge the funding support from the Natural Sciences and Engineering Research Council's Discovery Grants program. We would also like to acknowledge the Advanced Microscopy Facility at the University of Victoria. 


\section{REFERENCES}

1. Thomson, J.A., et al., Embryonic stem cell lines derived from human blastocysts. Science, 1998. 282(5391): p. 1145-7.

2. Takahashi, K. and S. Yamanaka, Induction of pluripotent stem cells from mouse embryonic and adult fibroblast cultures by defined factors. Cell, 2006. 126(4): p. 663-76.

3. $\quad \mathrm{Yu}$, J., et al., Induced pluripotent stem cell lines derived from human somatic cells. Science, 2007. 318(5858): p. 1917-20.

4. Ebert, A.D., et al., Induced pluripotent stem cells from a spinal muscular atrophy patient. Nature, 2009. 457(7227): p. 277-U1.

5. Turksen, K., Human Embryonic Stem Cells Handbook. Methods in Molecular Biology. 873.

6. Dimos, J.T., et al., Induced pluripotent stem cells generated from patients with ALS can be differentiated into motor neurons. Science, 2008. 321(5893): p. 1218-1221.

7. Marchetto, M.C.N., et al., A Model for Neural Development and Treatment of Rett Syndrome Using Human Induced Pluripotent Stem Cells. Cell, 2010. 143(4): p. 527-539.

8. Nori, S., et al., Grafted human-induced pluripotent stem-cell-derived neurospheres promote motor functional recovery after spinal cord injury in mice. Proceedings of the National Academy of Sciences of the United States of America, 2011. 108(40): p. 16825-16830.

9. Kobayashi, Y., et al., Pre-Evaluated Safe Human iPSC-Derived Neural Stem Cells Promote Functional Recovery after Spinal Cord Injury in Common Marmoset without Tumorigenicity. Plos One, 2012. 7(12).

10. Oki, K., et al., Human-induced pluripotent stem cells form functional neurons and improve recovery after grafting in stroke-damaged brain. Stem cells, 2012. 30(6): p. 1120-33.

11. Nutt, S.E., et al., Caudalized human iPSC-derived neural progenitor cells produce neurons and glia but fail to restore function in an early chronic spinal cord injury model. Experimental Neurology, 2013. 248: p. 491-503.

12. Qin, J., et al., Transplantation of human neuro-epithelial-like stem cells derived from induced pluripotent stem cells improves neurological function in rats with experimental intracerebral hemorrhage. Neuroscience letters, 2013. 548: p. 95-100.

13. Wang, A., et al., Induced pluripotent stem cells for neural tissue engineering. Biomaterials, 2011. 32(22): p. 5023-32.

14. Saadai, P., et al., Human induced pluripotent stem cell-derived neural crest stem cells integrate into the injured spinal cord in the fetal lamb model of myelomeningocele. Journal of Pediatric Surgery, 2013. 48(1): p. 158-163.

15. Fu, J.P., et al., Mechanical regulation of cell function with geometrically modulated elastomeric substrates (vol 7, pg 733, 2010). Nature Methods, 2011. 8(2): p. 184-184.

16. Pan, F., et al., Topographic effect on human induced pluripotent stem cells differentiation towards neuronal lineage. Biomaterials, 2013. 34(33): p. 8131-9.

17. Sun, Y.B. and J.P. Fu, Mechanobiology: a new frontier for human pluripotent stem cells. Integrative Biology, 2013. 5(3): p. 450-457.

18. Downing, T.L., et al., Biophysical regulation of epigenetic state and cell reprogramming. Nature materials, 2013. 12(12): p. 1154-62.

19. Zoldan, J., et al., The influence of scaffold elasticity on germ layer specification of human embryonic stem cells. Biomaterials, 2011. 32(36): p. 9612-9621.

20. Xie, J.W., et al., The differentiation of embryonic stem cells seeded on electrospun nanofibers into neural lineages. Biomaterials, 2009. 30(3): p. 354-362.

21. Travis Cordie, T.H., Xin Jing, Jared Carlson-Stevermer, Hao-Yang Mi, Lih-Sheng Turng, Krishanu Saha Nanofibrous Electrospun Polymers for Reprogramming Human Cells. Cellular and Molecular Bioengineering, 2014. 7(3): p. 379-393.

22. Cao, H., T. Liu, and S.Y. Chew, The application of nanofibrous scaffolds in neural tissue engineering. Advanced drug delivery reviews, 2009. 61(12): p. 1055-64. 
23. Subramanian, A., U.M. Krishnan, and S. Sethuraman, Development of biomaterial scaffold for nerve tissue engineering: Biomaterial mediated neural regeneration. Journal of biomedical science, 2009. 16: p. 108.

24. Madduri, S., M. Papaloizos, and B. Gander, Trophically and topographically functionalized silk fibroin nerve conduits for guided peripheral nerve regeneration. Biomaterials, 2010. 31(8): p. 2323-2334.

25. Xie, J., et al., Electrospun nanofibers for neural tissue engineering. Nanoscale, 2010. 2(1): p. 3544.

26. Mahairaki, V., et al., Nanofiber matrices promote the neuronal differentiation of human embryonic stem cell-derived neural precursors in vitro. Tissue engineering. Part A, 2011. 17(56): p. 855-63.

27. Jiang, X., et al., Nanofiber topography and sustained biochemical signaling enhance human mesenchymal stem cell neural commitment. Acta biomaterialia, 2012. 8(3): p. 1290-302.

28. Mohtaram, N., et al., Multifunctional Electrospun Scaffolds for Promoting Neuronal Differentiation of Induced Pluripotent Stem Cells. Journal of Biomaterials and Tissue Engineering, 2014. In press.

29. Mohtaram, N.K., A. Montgomery, and S.M. Willerth, Biomaterial-based drug delivery systems for the controlled release of neurotrophic factors. Biomedical materials, 2013. 8(2).

30. Dalton, P.D., et al., Direct in vitro electrospinning with polymer melts. Biomacromolecules, 2006. 7(3): p. 686-690.

31. Dalton, P.D., et al., Electrospinning of polymer melts: Phenomenological observations. Polymer, 2007. 48(23): p. 6823-6833.

32. Brown, T.D., P.D. Dalton, and D.W. Hutmacher, Direct Writing By Way of Melt Electrospinning. Advanced Materials, 2011. 23(47): p. 5651-+.

33. Ko, J., et al., Fabrication of poly (-caprolactone) microfiber scaffolds with varying topography and mechanical properties for stem cell-based tissue engineering applications. Journal of biomaterials science. Polymer edition, 2013.

34. Dalton, P.D., et al., Patterned melt electrospun substrates for tissue engineering. Biomedical materials, 2008. 3(3): p. 034109.

35. Detta, N., et al., Melt electrospinning of polycaprolactone and its blends with poly(ethylene glycol). Polymer International, 2010. 59(11): p. 1558-1562.

36. Baraam, Z., Litjens, Oostwaard,Brink, Laake, Lebrin, Kats, Hochstenbach, Passier, Sonnenberg and Mummery, Recombinant vitronectin is a functionally defined substrate that supports human embryonic stem cell self-renewal via alphavbeta5 integrin. Stem cells, 2008. 26(9): p. 2257-2265.

37. Ungrin, M.D., et al., Reproducible, ultra high-throughput formation of multicellular organization from single cell suspension-derived human embryonic stem cell aggregates. Plos One, 2008. 3(2): p. e1565.

38. Chen, G.K., et al., Chemically defined conditions for human iPSC derivation and culture. Nature Methods, 2011. 8(5): p. 424-U76.

39. Nakamura, M. and H. Okano, Cell transplantation therapies for spinal cord injury focusing on induced pluripotent stem cells. Cell research, 2013. 23(1): p. 70-80.

40. Willerth, S.M., et al., The effects of soluble growth factors on embryonic stem cell differentiation inside of fibrin scaffolds. Stem cells, 2007. 25(9): p. 2235-2244.

41. Ko, J., et al., Fabrication of poly (-caprolactone) microfiber scaffolds with varying topography and mechanical properties for stem cell-based tissue engineering applications. Journal of biomaterials science. Polymer edition, 2014. 25(1): p. 1-17.

42. Nagy, Z.K., et al., Solvent-free melt electrospinning for preparation of fast dissolving drug delivery system and comparison with solvent-based electrospun and melt extruded systems. Journal of Pharmaceutical Sciences, 2013. 102(2): p. 508-517. 
Table 1. Melt and solution electrospinning operational parameters. * In terms of bimodal scaffolds, all melt electrospinning parameters have been set as biaxial aligned scaffolds. Solution electrospinning parameters are given in the table for bimodal scaffolds.

\begin{tabular}{|l|c|c|c|c|}
\hline Parameters & $\begin{array}{c}\text { Loop Mesh 200 } \\
\text { Melt Electrospinning }\end{array}$ & $\begin{array}{c}\text { Loop Mesh 500 } \\
\text { Melt Electrospinning }\end{array}$ & $\begin{array}{c}\text { Biaxial Aligned } \\
\text { Melt Electrospinning }\end{array}$ & $\begin{array}{c}\text { Bimodal }^{*} \\
\text { Solution } \\
\text { Electrospinning }\end{array}$ \\
\hline Voltage $(\mathrm{kV})$ & 20 & 20 & 15 & 15 \\
\hline Distance $(\mathrm{cm})$ & 5 & 5 & 5 & N/A \\
\hline CNC Speed $(\mathrm{mm} / \mathrm{s})$ & 200 & 200 & 80 & 23 \\
\hline Temperature $\left({ }^{0} \mathrm{C}\right)$ & 80 & 80 & 200 & N/A \\
\hline Nozzle size $(\mu \mathrm{m})$ & 200 & 500 & & 1700 \\
\hline
\end{tabular}

Table 2. Micro and nanostructure topographical properties of scaffolds $(n=50)$. The average nanofiber diameter for bimodal scaffolds was $344.9 \pm 33.6 \mathrm{~nm}(n=100)$.

\begin{tabular}{|l|c|c|}
\hline Scaffold Type & Fiber Diameter \pm SD $(\mu \mathrm{m})$ & Separation Distance \pm SD $(\mu \mathrm{m})$ \\
\hline Loop Mesh 200 & $43.7 \pm 3.90$ & $177.9 \pm 106.4$ \\
\hline Loop Mesh 500 & $85 \pm 4$ & $141.1 \pm 54.2$ \\
\hline Biaxial Aligned $^{*}$ & $42.3 \pm 2.78$ & $161.1 \pm 99.2$ \\
\hline Bimodal $^{*}$ & $42.3 \pm 2.78$ & $161.1 \pm 99.2$ \\
\hline
\end{tabular}


Figure Legends

Figure 1. Scanning electron microscopy images of electrospun scaffolds. (A), (B) Low and high magnification images of loop mesh 200 scaffolds. (C), (D) Low and high magnification images of loop mesh 500 scaffolds. (E), (F) Low and high magnification images of biaxial aligned scaffolds fabricated with $200 \mu \mathrm{m}$ nozzle. (G) Low magnification image of bimodal scaffolds. (H) Retinoic acid encapsulated in poly (eta-caprolactone) nanofibers spun on top of biaxial aligned microfibers, resulting in novel bimodal scaffolds.

Figure 2. Neural progenitors seeded on loop mesh scaffolds after 12 days of culture. (A),(B) Bright field image and fluorescence image showing live and dead staining of cells seeded on loop mesh 200 scaffolds. (C), (D) Bright field image and fluorescence image showing staining for the neuronal marker Tuj1 expressed by cells seeded on loop mesh 200 scaffolds. (E), (F) Bright field image and fluorescence image showing live and dead staining of cells seeded on the loop mesh 500 scaffold. (G), (H) Bright field image and fluorescence image showing staining for the neuronal marker Tuj1 expressed by cells seeded on loop mesh 500 scaffolds.

Figure 3. Neural progenitors seeded on biaxial aligned scaffolds fabricated using a $200 \mu \mathrm{m}$ nozzle after 12 days of culture. (A),(B) Bright field image and fluorescence image showing live and dead staining of cells. (C), (D) Bright field image and fluorescence image showing staining for the neuronal marker Tuj1 expressed by cells.

Figure 4. Neural progenitors seeded on bimodal scaffolds after 12 days of culture. (A), (B) Bright field image and fluorescence image showing live and dead staining of cells seeded on bimodal scaffolds. (C), (D) Bright field image and fluorescence image showing neuronal marker Tuj1 staining for two adjacent neural aggregates seeded on bimodal scaffolds that have neuronal interconnections.

Figure 5. Quantitative analysis of cell viability and differentiation when seeded on electrospun scaffolds. (A) Mean intensity of green fluorescence. (B) Cell body cluster area. (C) Maximum neurite length. * indicates $\mathrm{p}<0.05$ versus other scaffolds. \# indicates $\mathrm{p}<0.05$ for loop mesh 200 scaffolds compared to loop mesh 500 scaffolds. $\mathrm{N}=3$.

Figure 6. Quantitative analysis of gene expression in human iPSC-derived neural progenitors cultured on scaffolds for 12 days. The markers examined using quantitative polymerase chain reaction (qPCR) were Oct4, Lin28, Nestin, Pax6. * indicates p $<0.05$ versus other scaffolds. \# indicates $\mathrm{p}<0.05$ for loop mesh 200 scaffolds compared to loop mesh 500 scaffolds. + indicates $\mathrm{p}<0.05$ for biaxial aligned scaffolds compared to bimodal scaffolds. All expression levels are normalized relative to undifferentiated human iPSCs as control. $\mathrm{N}=3$. 

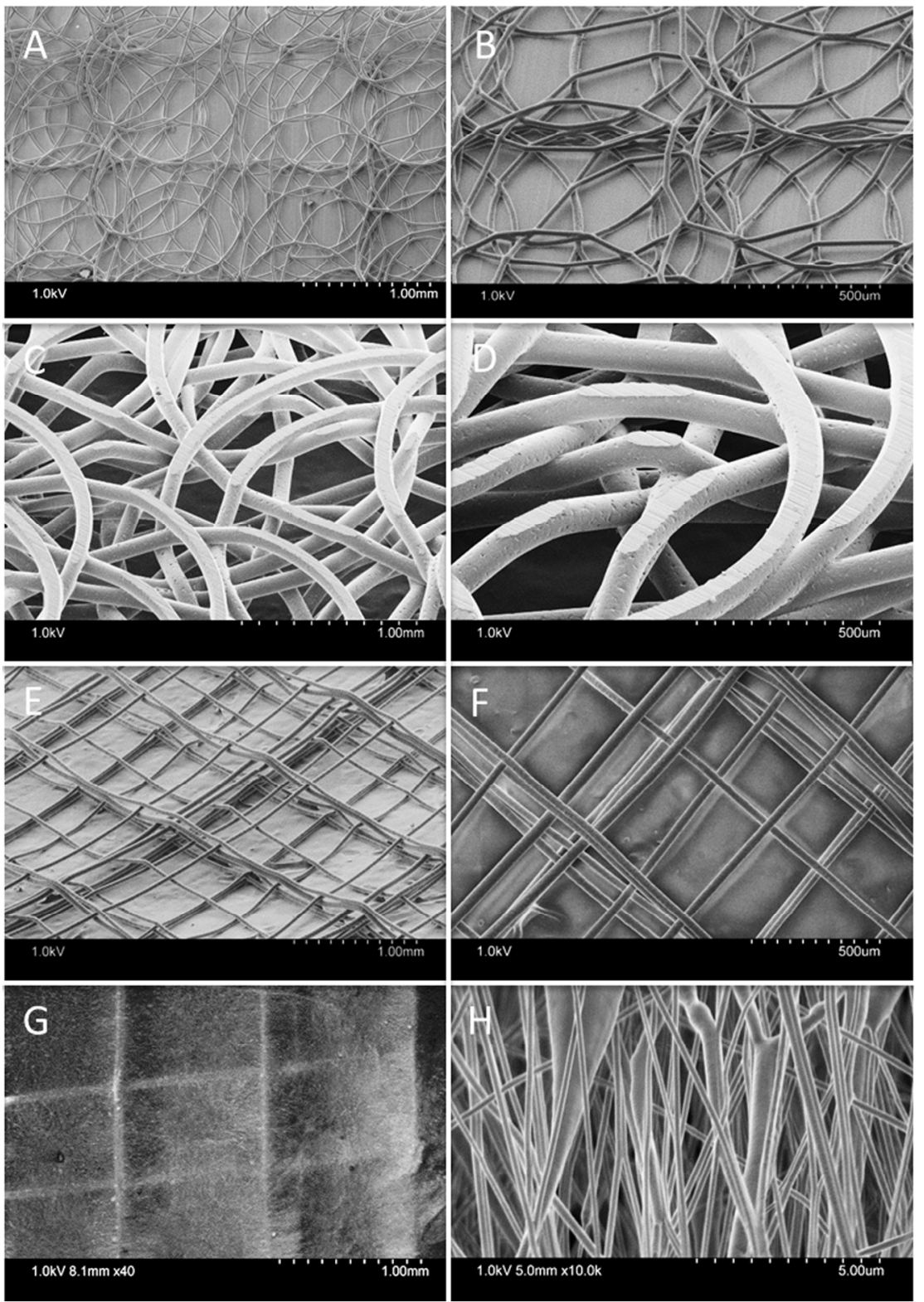

Figure 1. Scanning electron microscopy images of electrospun scaffolds. (A), (B) Low and high magnification images of loop mesh 200 scaffolds. (C), (D) Low and high magnification images of loop mesh 500 scaffolds.

(E), (F) Low and high magnification images of biaxial aligned scaffolds fabricated with $200 \mu \mathrm{m}$ nozzle. (G) Low magnification image of bimodal scaffolds. $(H)$ Retinoic acid encapsulated in poly (eta-caprolactone) nanofibers spun on top of biaxial aligned microfibers, resulting in novel bimodal scaffolds. $198 \times 281 \mathrm{~mm}(300 \times 300 \mathrm{DPI})$ 


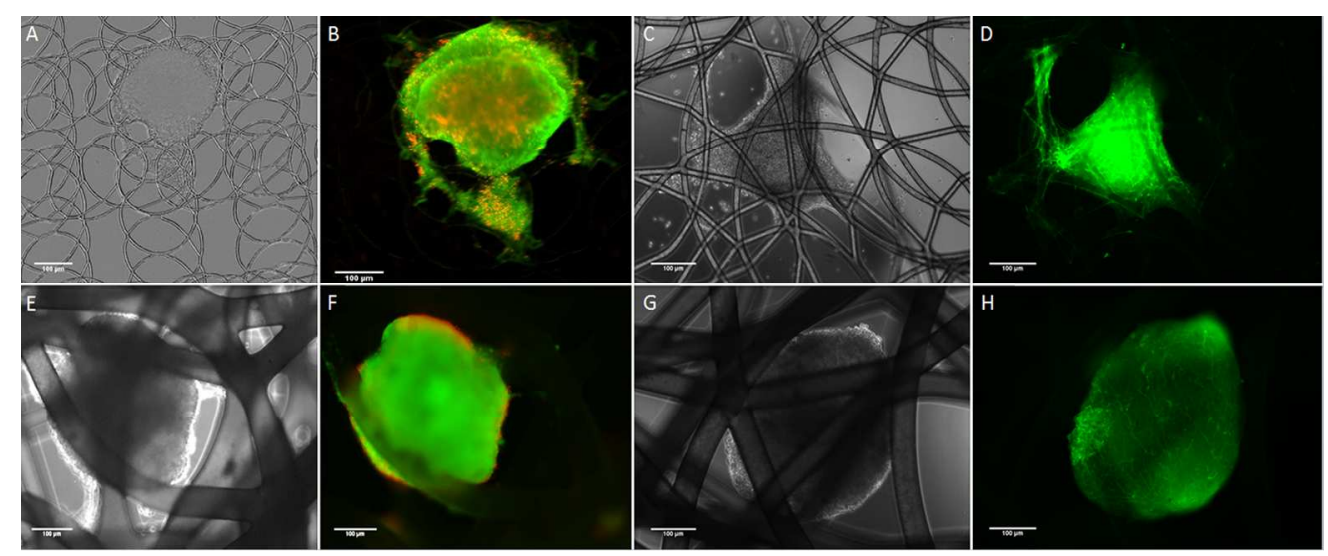

Figure 2. Neural progenitors seeded on loop mesh scaffolds after 12 days of culture. (A),(B) Bright field image and fluorescence image showing live and dead staining of cells seeded on loop mesh 200 scaffolds. (C), (D) Bright field image and fluorescence image showing staining for the neuronal marker Tuj1 expressed by cells seeded on loop mesh 200 scaffolds. (E), (F) Bright field image and fluorescence image showing live and dead staining of cells seeded on the loop mesh 500 scaffold. $(G),(H)$ Bright field image and fluorescence image showing staining for the neuronal marker Tuj1 expressed by cells seeded on loop mesh 500 scaffolds. $415 \times 170 \mathrm{~mm}(300 \times 300 \mathrm{DPI})$ 


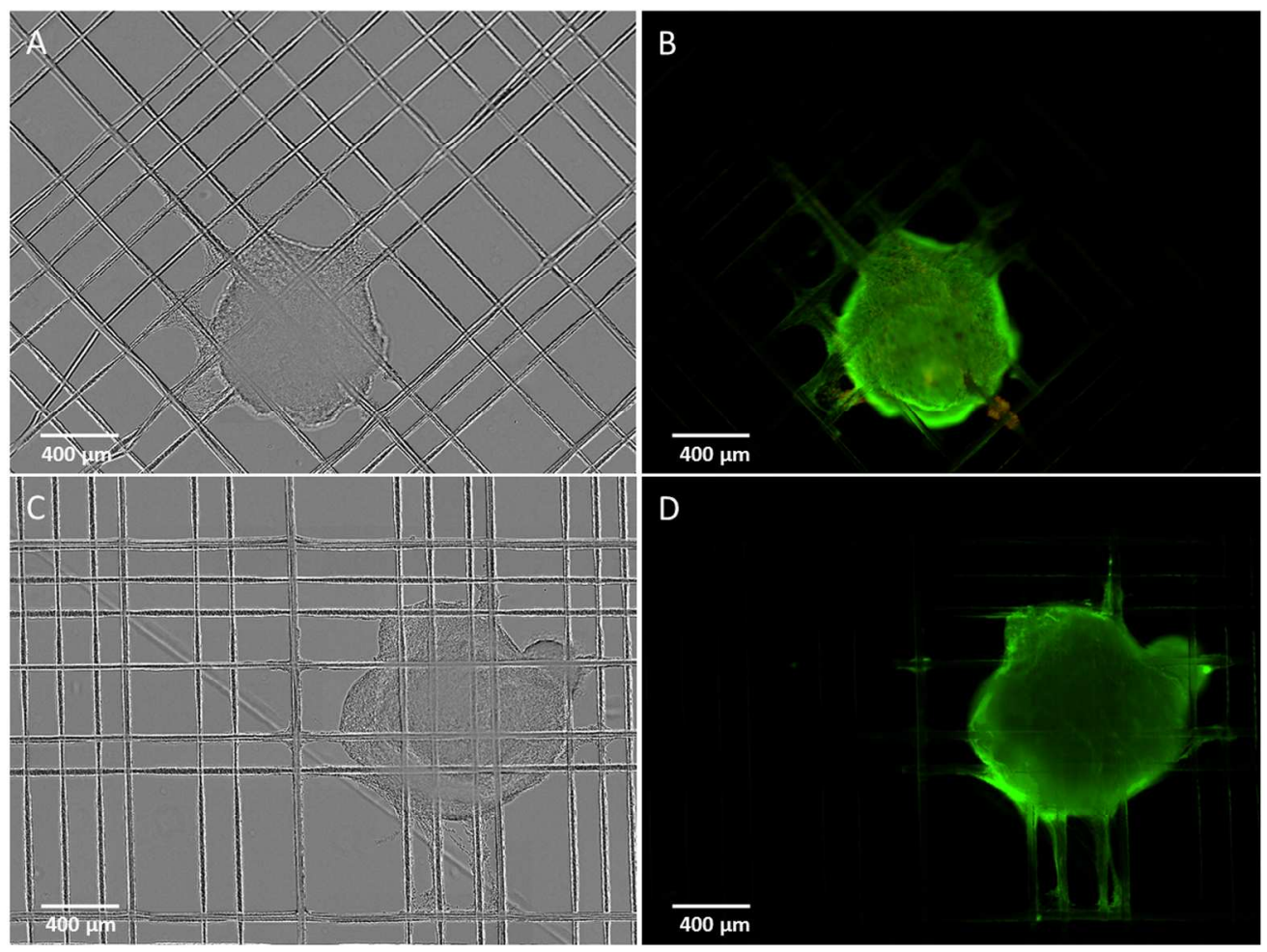

Figure 3. Neural progenitors seeded on biaxial aligned scaffolds fabricated using a $200 \mu \mathrm{m}$ nozzle after 12 days of culture. (A),(B) Bright field image and fluorescence image showing live and dead staining of cells. (C), (D) Bright field image and fluorescence image showing staining for the neuronal marker Tuj1 expressed by cells. $324 \times 242 \mathrm{~mm}(300 \times 300 \mathrm{DPI})$ 

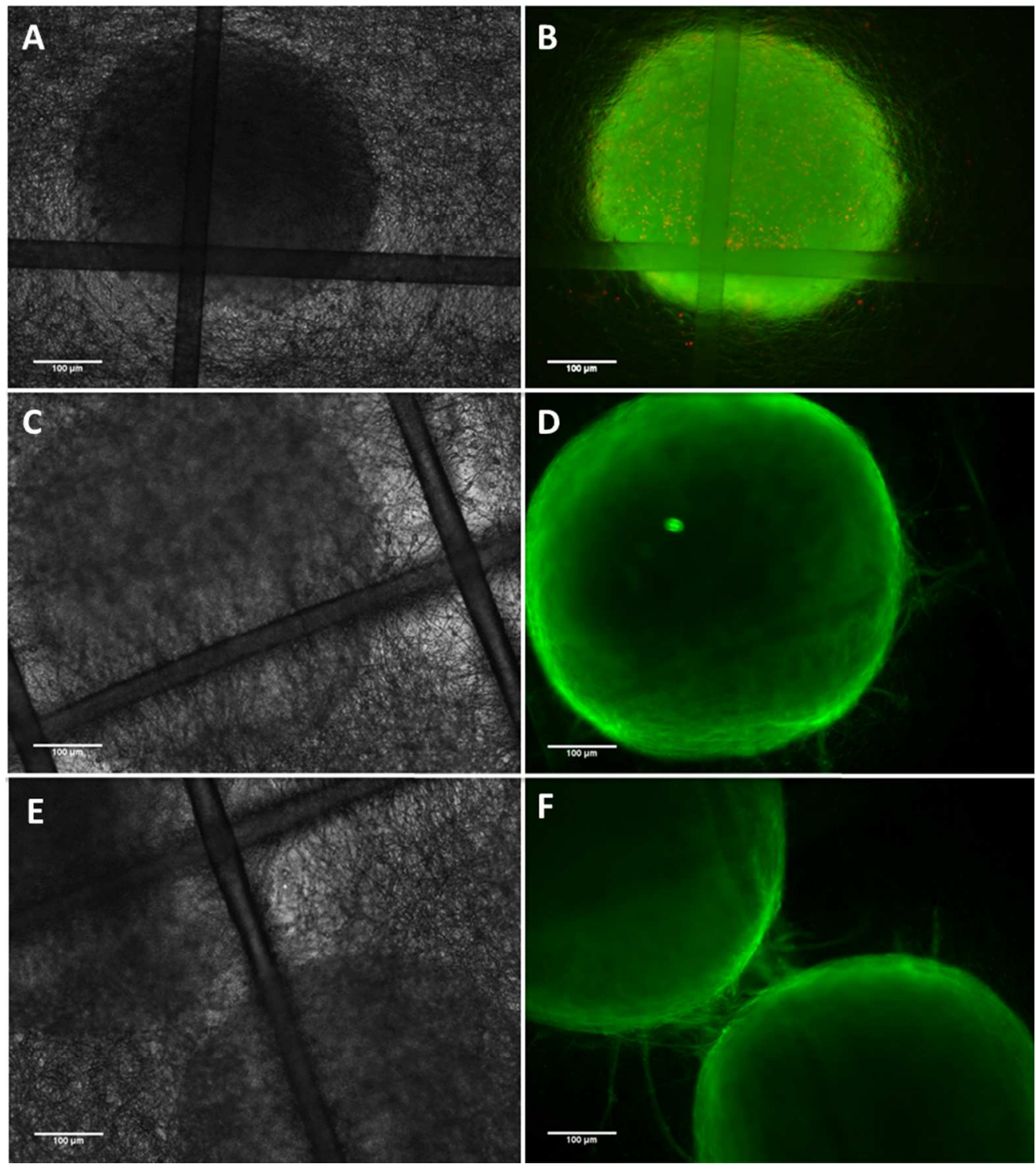

F

Figure 4. Neural progenitors seeded on bimodal scaffolds after 12 days of culture. (A), (B) Bright field image and fluorescence image showing live and dead staining of cells seeded on bimodal scaffolds. (C), (D) Bright field image and fluorescence image showing neuronal marker Tuj1 staining for two adjacent neural aggregates seeded on bimodal scaffolds that have neuronal interconnections. $253 \times 285 \mathrm{~mm}(300 \times 300 \mathrm{DPI})$ 

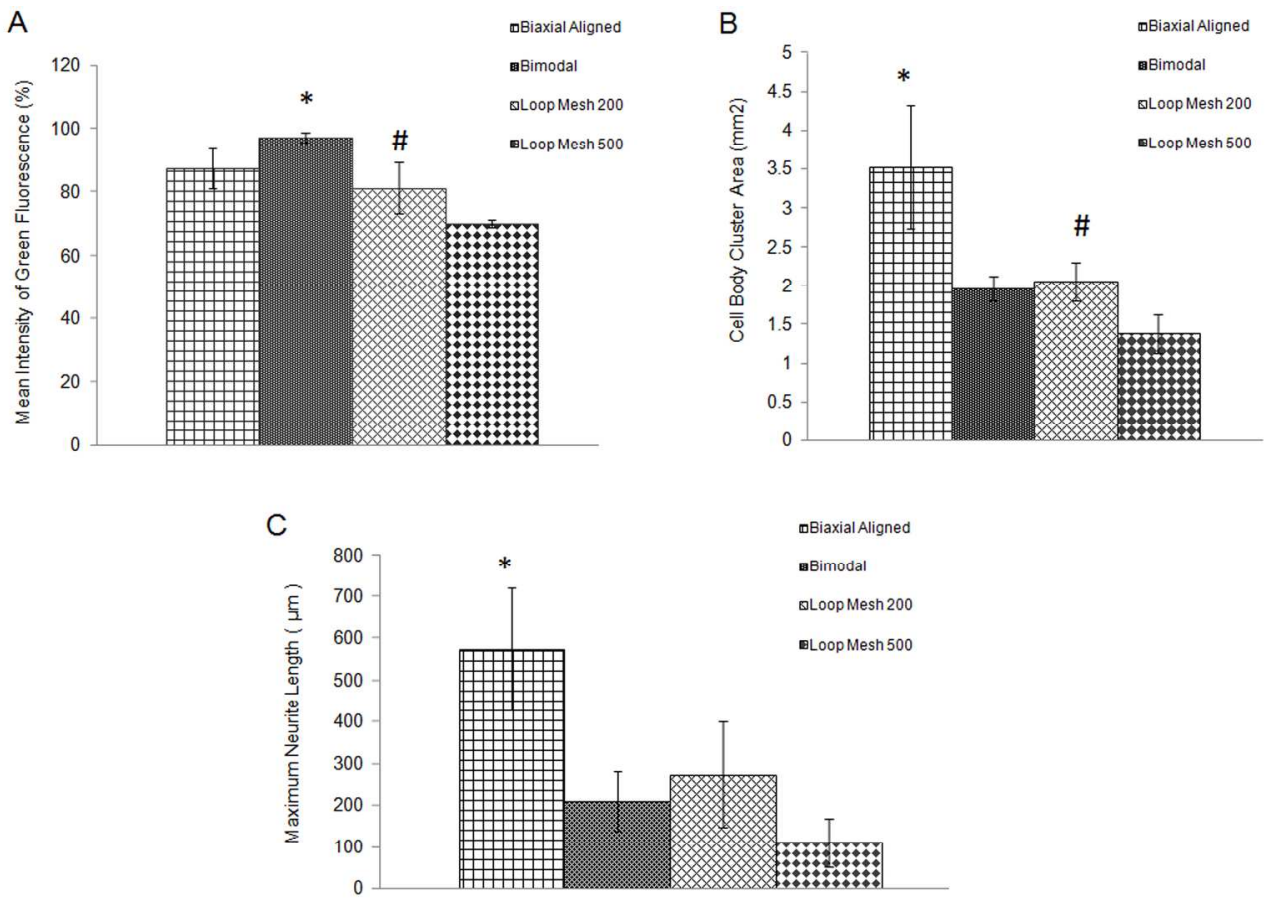

Figure 5. Quantitative analysis of cell viability and differentiation when seeded on electrospun scaffolds. (A) Mean intensity of green fluorescence. (B) Cell body cluster area. (C) Maximum neurite length. $*$ indicates $p<0.05$ versus other scaffolds. \# indicates $p<0.05$ for loop mesh 200 scaffolds compared to loop mesh 500 scaffolds. $\mathrm{N}=3$.

$207 \times 155 \mathrm{~mm}(300 \times 300 \mathrm{DPI})$

John Wiley \& Sons, Inc. 
Figure 6. Quantitative analysis of gene expression in human iPSC-derived neural progenitors cultured on scaffolds for 12 days. The markers examined using quantitative polymerase chain reaction (qPCR) were Oct4, Lin28, Nestin, Pax6. * indicates $p<0.05$ versus other scaffolds. \# indicates $p<0.05$ for loop mesh 200 scaffolds compared to loop mesh 500 scaffolds. + indicates $p<0.05$ for biaxial aligned scaffolds compared to bimodal scaffolds. All expression levels are normalized relative to undifferentiated human iPSCs as control. $\mathrm{N}=3$.

$207 \times 155 \mathrm{~mm}(300 \times 300 \mathrm{DPI})$ 
Table 1. Melt and solution electrospinning operational parameters. * In terms of bimodal scaffolds, all melt electrospinning parameters have been set as biaxial aligned scaffolds. Solution electrospinning parameters are given in the table for bimodal scaffolds.

\begin{tabular}{|l|c|c|c|c|}
\hline Parameters & $\begin{array}{c}\text { Loop Mesh 200 } \\
\text { Melt Electrospinning }\end{array}$ & $\begin{array}{c}\text { Loop Mesh 500 } \\
\text { Melt Electrospinning }\end{array}$ & $\begin{array}{c}\text { Biaxial Aligned } \\
\text { Melt Electrospinning }\end{array}$ & $\begin{array}{c}\text { Bimodal }^{*} \\
\text { Solution } \\
\text { Electrospinning }\end{array}$ \\
\hline Voltage $(\mathrm{kV})$ & 20 & 20 & 15 & 15 \\
\hline Distance $(\mathrm{cm})$ & 5 & 5 & 5 & 7.5 \\
\hline CNC Speed $(\mathrm{mm} / \mathrm{s})$ & 200 & 200 & 1700 & N/A \\
\hline Temperature $\left({ }^{0} \mathrm{C}\right)$ & 80 & 80 & 80 & 23 \\
\hline Nozzle size $(\mu \mathrm{m})$ & 200 & 500 & 200 & N/A \\
\hline
\end{tabular}

Table 2. Micro and nanostructure topographical properties of scaffolds $(n=50) . *$ The average nanofiber diameter for bimodal scaffolds was $344.9 \pm 33.6 \mathrm{~nm}(n=100)$.

\begin{tabular}{|l|c|c|}
\hline Scaffold Type & Fiber Diameter \pm SD $(\mu \mathrm{m})$ & Separation Distance \pm SD $(\mu \mathrm{m})$ \\
\hline Loop Mesh 200 & $43.7 \pm 3.90$ & $177.9 \pm 106.4$ \\
\hline Loop Mesh 500 & $85 \pm 4$ & $141.1 \pm 54.2$ \\
\hline Biaxial Aligned & $42.3 \pm 2.78$ & $161.1 \pm 99.2$ \\
\hline Bimodal $^{*}$ & $42.3 \pm 2.78$ & $161.1 \pm 99.2$ \\
\hline
\end{tabular}

